\title{
Photon emission from quark-gluon plasma out of equilibrium
}

\author{
Sigtryggur Hauksson ${ }^{*}$ Sangyong Jeon, and Charles Gale \\ Department of Physics, McGill University, 3600 University Street, Montreal, QC, H3A 2T8, Canada
}

\begin{abstract}
The photon emission from a non-equilibrium quark-gluon plasma (QGP) is analyzed. We derive an integral equation that describes photon production through quark-antiquark annihilation and quark bremsstrahlung. It includes coherence between different scattering sites, also known as the LandauPomeranchuk-Migdal effect. These leading-order processes are studied for the first time together in an out-of-equilibrium field theoretical treatment that enables the inclusion of viscous corrections to the calculation of electromagnetic emission rates. In the special case of an isotropic, viscous, plasma the integral equation only depends on three constants which capture the non-equilibrium nature of the medium.
\end{abstract}

\section{INTRODUCTION}

Relativistic collisions of large nuclei allow the study of QCD (Quantum Chromodynamics: the theory of the nuclear strong interaction) matter at high temperatures. Experiments performed at the Relativistic Heavy Ion Collider (RHIC) and the Large Hadron Collider (LHC) have indeed shown that such collisions create droplets of quark-gluon plasma (QGP) [1]. A significant breakthrough in the relativistic heavy-ion program has been the realization that this fluid and its evolution can be characterized by relativistic hydrodynamics, which describes long-wavelength excitations [2]. Therefore, those experiments have the potential to give access to the transport coefficients of QGP, such as shear and bulk viscosity. These coefficients are fundamental properties of QCD.

There has been extensive work on extracting the viscosity of QGP from soft hadronic observables 3]. In addition, electromagnetic observables, i.e. photons and dileptons, can play an important role in that endeavour [4, 5. They are emitted throughout the evolution of the QGP and escape from the medium unaffected by final state interaction. This work will focus on the production of real photons.

In order to evaluate the effects of viscosity on photons one must study their out-of-equilibrium emission. At leading order in the strong coupling constant there are two channels for photon production in QGP. Firstly, there are two-to-two scattering channels with a photon in the final state. They were first calculated in [6, 7] for thermal equilibrium. Since then there have been a number of works on these processes in an out-of-equilibrium QGP such as for finite fugacities [8 10, in an anisotropic QGP [11, for shear viscous corrections [12, 13] and for bulk viscous corrections 14 . Note that consistent calculations of the electromagnetic emissivity should include non-equilibrium corrections to the thermal mass of the soft mediators.

Secondly, there are inelastic channels with bremsstrahlung off a quark and the pair annihilation of a quark and antiquark, see Fig. 11. These

\footnotetext{
* Corresponding author: sigtryggur.hauksson@mail.mcgill.ca
}
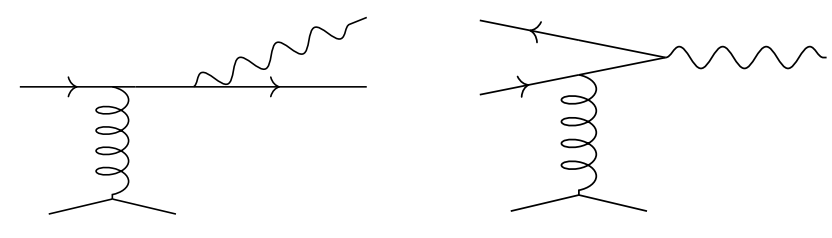

FIG. 1. Photon production through quark bremsstrahlung and the annihilation of a quark and an antiquark. For the first process, the diagram with the photon emitted left of the vertex is included but not shown, as is that with the gluon connecting to the antiquark in the second process.

processes contribute as much to photon emission as two-to-two scattering [15. The photon is emitted almost collinearily to the quark, which entails a large decoherence time. This means that the quarks can exchange arbitrarily many soft gluons with the medium during the formation of the photon. This leading-order complication is known as the Landau-PomeranchukMigdal (LPM) effect [16 19]. It means that in addition to the diagrams in Fig. 1 one must sum up diagrams with an arbitrary number of gluon exchanges such as in Fig. 4. The LPM effect was first treated consistently in [20, 21] for a medium in thermal equilibrium where it was shown to reduce photon production because of coherence between different emission sites.

This work treats the photon production through inelastic channels in a non-equilibrium QGP for the first time, using a field theoretical derivation which includes the LPM effect without relying on the Kubo-MartinSchwinger (KMS) 22 relation which describes detailed balance in thermal equilibrium. The field-theoretical techniques used here differ from those employed in Ref. [23, where kinetic theory was used to analyze inelastic scattering of quarks and gluons.

The paper is organized as follows. In Sec. II] we review the real-time formalism and derive expressions for resummed propagators. In Sec. III we discuss the resummed $r r$ propagator for soft gluons and show that the LPM effect is leading order in non-equilibrium systems. Sec. IV] discusses the resummed occupation number of hard quarks. In Sec. $\mathrm{V}$ we sum up the different diagrams contributing to the LPM effect and derive an inte- 

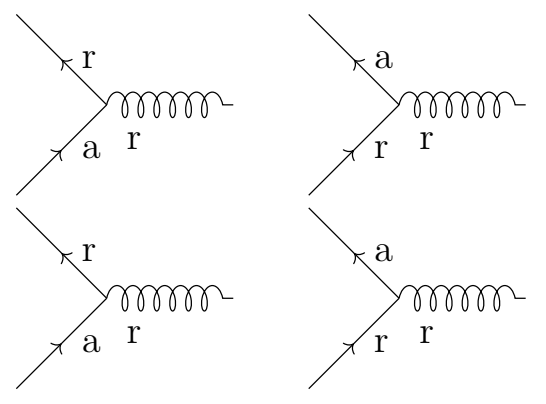

FIG. 2. Quark-gluon vertices in the $r / a$ basis in the real time formalism

gral equation describing the inelastic channels. Finally, we conclude in Sec. VI and indicate future directions. We will use the $(+,-,-,-)$ metric. If $P^{\mu}$ is a four-vector we write $P^{\mu}=\left(p^{0}, \mathbf{p}\right)$ and define $p=|\mathbf{p}|$ and $\hat{\mathbf{p}}=\mathbf{p} / p$.

\section{RESUMMED PROPAGATORS IN THE REAL-TIME FORMALISM}

Out-of-equilibrium quantum field theorie is best described in the real-time formalism where a closed time contour leads to the doubling of degrees of freedom [24, 25]. In this paper we mostly work in the $r / a$ basis 26] which is defined by

$$
\phi_{r}=\frac{1}{2}\left(\phi_{1}+\phi_{2}\right), \quad \phi_{a}=\phi_{1}-\phi_{2} .
$$

The propagators are

$$
D_{c d}(x, y)=\left\langle\phi_{c}(x) \phi_{d}^{\dagger}(y)\right\rangle=\operatorname{Tr}\left[\rho_{0} \mathcal{T}_{\mathcal{C}} \phi_{c}(x) \phi_{d}^{\dagger}(y)\right]
$$

where $c$ and $d$ are either $r$ or $a$. The initial density matrix $\rho_{0}$ determines the out-of-equilibrium evolution of the system. In the $r / a$ basis, vertices have an odd number of $a$ indices, see Fig. 2. This basis has numerous advantages: The $a a$ propagator vanishes identically and the bare $r a$ and ar propagators only include vacuum contributions. Furthermore it allows for easier power counting. For concreteness we consider complex scalar fields in this section but our arguments can easily be generalized.

In this paper we study translationally invariant systems. In other words, our calculation is for a homogeneous and static brick of out-of-equilibrium QGP. This approximation is justified when the mean free path of quasiparticles is much smaller scale than the macroscopic scale at which hydrodynamical quantities change appreciably. In a translationally invariant system the propagators are

$$
\begin{aligned}
& D_{r r}(x)=\frac{1}{2}\left\langle\left\{\phi(x), \phi^{\dagger}(0)\right\}\right\rangle \\
& D_{r a}(x)=\theta\left(x^{0}\right)\left\langle\left[\phi(x), \phi^{\dagger}(0)\right]\right\rangle \\
& D_{a r}(x)=-\theta\left(-x^{0}\right)\left\langle\left[\phi(x), \phi^{\dagger}(0)\right]\right\rangle \\
& D_{a a}(x)=0 .
\end{aligned}
$$

We see that $D_{r a}=D_{\text {ret }}$ and $D_{a r}=D_{\text {adv }}$. Going to momentum space with momentum $P$

$$
\begin{aligned}
D_{\text {ret }}(P)^{*} & =\int d^{4} x e^{-i P \cdot x} \theta\left(x^{0}\right)\left\langle\left[\phi(0), \phi^{\dagger}(x)\right]\right\rangle \\
& =\int d^{4} x e^{i P \cdot x} \theta\left(-x^{0}\right)\left\langle\left[\phi(0), \phi^{\dagger}(-x)\right]\right\rangle \\
& =\int d^{4} x e^{i P \cdot x} \theta\left(-x^{0}\right)\left\langle\left[\phi(x), \phi^{\dagger}(0)\right]\right\rangle \\
& =-D_{\text {adv }}(P)
\end{aligned}
$$

where we did a change of variables $x \rightarrow-x$ in the second line and then used translational invariance. This shows that the resummed propagators only have two independent components. The bare retarded propagator can easily be evaluated because the commutator of free bosonic fields is just a number so summing over all states is trivial. It is the same as in vacuum,

$$
D_{\text {ret }}^{0}(P)=\frac{i}{P^{2}+i \epsilon p^{0}}
$$

We have yet to find the $r r$ propagator. In thermal equilibrium the Kubo-Martin-Schwinger (KMS) relation stipulates that

$$
D_{r r}(P)=\left(\frac{1}{2}+f_{B}\left(p^{0}\right)\right)\left[D_{\mathrm{ret}}(P)-D_{\mathrm{adv}}(P)\right]
$$

so there is only one independent propagator. Here $f_{B}\left(p^{0}\right)$ is the Bose-Einstein distribution [24]. This expression is valid at every order in perturbation theory and thus offers great simplification. Using Eq. (5) the bare $r r$ propagator is

$$
D_{r r}^{0}(P)=\left(\frac{1}{2}+f_{B}(p)\right) 2 \pi \delta\left(P^{2}\right) .
$$

In non-equilibrium systems one cannot obtain a general expression for the resummed $r r$ propagator. In analogy with the equilibrium case the bare propagator is

$$
D_{r r}^{0}(P)=\left(\frac{1}{2}+\theta\left(p^{0}\right) f(\mathbf{p})+\theta\left(-p^{0}\right) f(-\mathbf{p})\right) 2 \pi \delta\left(P^{2}\right)
$$

where the ansatz, $f(\mathbf{p})$, is a general momentum distribution characterizing the system. For mirror symmetric momentum distributions, $f(\mathbf{p})=f(-\mathbf{p})$, the bracket reduces to $1 / 2+f(\mathbf{p})$, see [27] for a discussion. Eq. (8) can be justified from first principles as in 28, 29. Assuming an initial density matrix $\rho_{0}$ one Legendre-transforms the path integral from external sources to connected n-point functions. This gives rise to an infinite tower of equations corresponding to the BBGKY hierarchy in kinetic theory. Truncating the tower at second order and assuming that propagators vary slowly in space one gets Eq. (8) at lowest order in the coupling. The function $f$ can be shown to be real and positive and the equations of motion reduce to a Boltzmann equation for $f$. Therefore, at leading order in the coupling constant it should be interpreted as a 
momentum distribution of particles. The delta function shows that the quasiparticles are on shell to lowest order. In this approach $f$ is left unspecified and can be chosen to match a hydrodynamical evolution of the QGP.

Demanding that propagators vary slowly in time sets constraints on how far from equilibrium one can go. In anisotropic and translationally invariant systems the retarded gluon propagator acquires a pole with $\operatorname{Im} \omega>0$ 30. This pole signals the exponential growth of the occupation density of soft gluons [31] which can invalidate our assumption of translational invariance. Specifically, the pole introduces divergences in momentum integrals over $G_{r r}$ for soft gluons. Throughout our analysis we will assume that the anisotropy is small enough so that this divergence does not appear at leading order in the coupling. As an example consider a momentum distribution of the form 30

$$
f(\mathbf{p})=f_{\mathrm{eq}}\left(\sqrt{p^{2}+\xi(\mathbf{n} \cdot \mathbf{p})^{2}}\right)
$$

where $f_{\text {eq }}$ is an equilibrium distribution and $\mathbf{n}$ is a unit vector specifying the direction of the anisotropy $\xi$ (in general $-1 \leq \xi<\infty)$. We will show below that we must demand $|\xi| \lesssim g^{2}$ if the divergence is to be subleading. On the contrary, isotropic systems can be much further away from equilibrium without our analysis breaking down. In summary, we study systems with low anisotropy that are close enough to local thermal equilibrium so that the equilibrium power counting scheme is unaltered. This guarantees that the hard thermal loop (HTL) scheme remains valid.

In this paper we will need resummed propagators in out-of-equilibrium systems. For the convenience of the reader we reproduce some known results for scalar field theory in the real-time formalism, see [32. The Dyson equation is

$$
\left[\begin{array}{ll}
D_{r r} & D_{r a} \\
D_{a r} & D_{a a}
\end{array}\right]=\left[\begin{array}{ll}
D_{r r}^{0} & D_{r a}^{0} \\
D_{a r}^{0} & D_{a a}^{0}
\end{array}\right]+\left[\begin{array}{cc}
D_{r r}^{0} & D_{r a}^{0} \\
D_{a r}^{0} & D_{a a}^{0}
\end{array}\right](-i)\left[\begin{array}{ll}
\Pi_{r r} & \Pi_{r a} \\
\Pi_{a r} & \Pi_{a a}
\end{array}\right]\left[\begin{array}{ll}
D_{r r} & D_{r a} \\
D_{a r} & D_{a a}
\end{array}\right]
$$

where, say, $\Pi_{a a}$ is the self-energy sourced by two $a$ fields. Using $D_{a a}=D_{a a}^{0}=0$ one finds that $\Pi_{r r}=0$. Defining $\Pi_{a r}=\Pi_{\text {ret }}$ one obtains

$$
D_{\text {ret }}=D_{\text {ret }}^{0}+D_{\text {ret }}^{0}\left(-i \Pi_{\text {ret }}\right) D_{\text {ret }}
$$

which gives

$$
D_{\mathrm{ret}}=\frac{i}{P^{2}-\Pi_{\mathrm{ret}}}
$$

This equation gives the dispersion relation for the quasiparticles, i.e. their thermal mass and decay width. Sim- ilarly

$$
D_{\mathrm{adv}}=\frac{i}{P^{2}-\Pi_{\mathrm{adv}}}
$$

where $\Pi_{\mathrm{adv}}=\Pi_{r a}=\Pi_{\mathrm{ret}}^{*}$ in translationally invariant systems.

The Dyson equation for $D_{r r}$ is more complicated. Defining $D_{<}=D_{12}$ and $D_{>}=D_{21}$ we can write

$$
D_{r r}=\frac{1}{2}\left(D_{>}+D_{<}\right)=D_{<}+\frac{1}{2}\left(D_{\text {ret }}-D_{\text {adv }}\right)
$$

since $D_{>}-D_{<}=D_{\text {ret }}-D_{\text {adv }}$. We will analyze $D_{<}$ to obtain equations with a clear physical interpretation. Using the $r r$ component of Eq. 10, Eq. (11) and the corresponding equation for $D_{\text {adv }}$ one gets

$$
D_{<}=D_{<}^{0}+D_{\text {ret }}^{0}\left(-i \Pi_{\text {ret }}\right) D_{<}+D_{<}^{0}\left(-i \Pi_{\text {adv }}\right) D_{\text {adv }}+D_{\text {ret }}^{0}\left(-i \Pi_{<}\right) D_{\text {adv }}
$$

where

one gets

$$
\Pi_{<}=\Pi_{a a}-\frac{1}{2} \Pi_{\mathrm{ret}}+\frac{1}{2} \Pi_{\mathrm{adv}}
$$

In the original 12 basis $\Pi_{<}=-\Pi_{12}$ in our convention. This component of the self-energy describes the creation rate of quasi-particles [24]. Solving for $D_{<}$using Eq. [12]

For non-vanishing self-energy the first term is zero be- 
cause

$$
D_{<}^{0}\left(D_{\text {ret }}^{0}\right)^{-1} \propto P^{2} \delta\left(P^{2}\right)=0
$$

This is true since our theory is defined in momentum space and assumes translational invariance. Thus

$$
D_{<}=D_{\text {ret }}\left(-i \Pi_{<}\right) D_{\text {adv }}
$$

and

$$
D_{r r}=\frac{1}{2}\left(D_{\text {ret }}-D_{\text {adv }}\right)+D_{\text {ret }}\left(-i \Pi_{<}\right) D_{\text {adv }}
$$

In deriving this equation we never had to invert the order of propagators. Thus it is equally valid for spinors and spin- 1 bosons whose propagators are matrices in the spacetime indices.

For $D_{>}=D_{21}$ one similarly gets that

$$
D_{>}=D_{\text {ret }}\left(-i \Pi_{>}\right) D_{\mathrm{adv}}
$$

where $\Pi_{>}=-\Pi_{21}$ describes the annihilation of quasiparticles. It's easy to see that

$$
\Pi_{\mathrm{ret}}-\Pi_{\mathrm{adv}}=\Pi_{>}-\Pi_{<}
$$

which reduces to

$$
2 i \operatorname{Im} \Pi_{\text {ret }}=\Pi_{>}-\Pi_{<}
$$

in translationally invariant systems. This last equation says that the decay width of a quasi-particle is the difference of the annihilation and creation rate.

For scalar particles we can go further and derive a more intuitive expression for $D_{r r}$. In translationally invariant systems Eq. 12 and 13 give that

$$
D_{\text {ret }}-D_{\text {adv }}=2\left(\operatorname{Im} \Pi_{\text {ret }}\right) D_{\text {ret }} D_{\text {adv }} .
$$

Thus we see that

$$
D_{r r}=\left[\frac{1}{2}+\frac{\Pi_{<}}{2 i \operatorname{Im} \Pi_{\mathrm{ret}}}\right]\left(D_{\mathrm{ret}}-D_{\mathrm{adv}}\right) .
$$

This equation has a striking resemblance with the $r r$ propagator in equilibrium, Eq. (6). Indeed $\Pi_{<} / 2 i \operatorname{Im} \Pi_{\text {ret }}$ reduces to the Bose-Einstein distribution by using the KMS relation for self-energies. In non-equilibrium systems $\Pi_{<} / 2 i \operatorname{Im} \Pi_{\text {ret }}$ is in general not the same as the bare momentum distribution $f(\mathbf{p})$. It can be viewed as a resummed occupation density. We emphasize that we have only derived Eq. 25) for scalar particles since we needed to invert the order of propagators. In the next two sections we will derive a similar relation for soft gluons and hard quarks and evaluate the resummed occupation density explicitly.

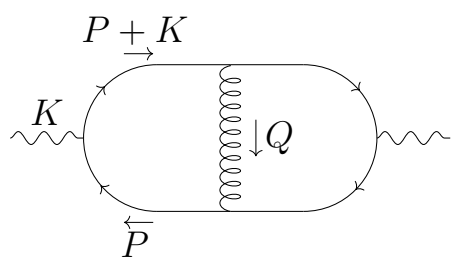

FIG. 3. Definition of momenta in the argument for bremsstrahlung and pair annihilation contribution at leading order.

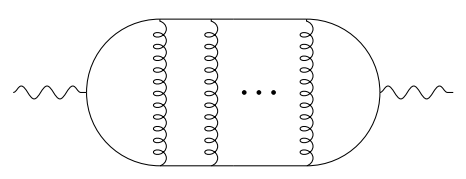

FIG. 4. The diagrams for the LPM effect.

\section{THE $r r$ PROPAGATOR OF SOFT GLUONS}

The photon production rate is given by the 12 component of the photon polarization tensor

$$
k \frac{d R}{d^{3} k}=\frac{i}{2(2 \pi)^{3}}\left(\Pi_{12}^{\gamma}\right)_{\mu}^{\mu},
$$

where $\mathbf{k}$ is the photon momentum and $\Pi_{12}^{\gamma}$ is one component of the photon polarization tensor. This equation is valid in non-equilibrium systems as has been shown in [33.

The diagram corresponding to bremsstrahlung and quark-antiquark pair annihilation is in Fig. 3. Due to the LPM effect the quarks can have arbitrarily many gluon exchanges, see Fig. 4. We will now briefly explain why these diagrams contribute at leading order for a medium in thermal equilibrium, see [20, 34] for further details. The quarks are hard, $P \sim T$, and nearly on shell, $P^{2} \sim g^{2} T^{2}$, where $T$ is the temperature and $g \ll 1$ is the strong coupling constant. The photon is emitted with an angle $\theta \sim g$ relative to the quark momentum. Finally, the exchanged gluons are soft, $Q \sim g T$, forcing us to use resummed propagators.

We analyze the diagram in Fig. 3. In thermal equilibrium the $r r$ propagator for soft gluons is

$$
G_{r r}(Q)=\left(\frac{1}{2}+f_{B}\left(q^{0}\right)\right)\left[G_{\mathrm{ret}}-G_{\mathrm{adv}}\right] \sim \frac{1}{g^{3} T^{2}}
$$

where $f_{B}\left(q^{0}\right) \sim T / q^{0} \sim 1 / g$ and the retarded gluon propagator is $G_{\text {ret }} \sim 1 / g^{2} T^{2}$. Furthermore, each pair of quark propagators gives pinching poles of order $1 / g^{2}$. This can 
be seen more easily for bare scalars for which

$$
\begin{aligned}
\int d p^{0} & D_{a r}(K+P) D_{r a}(P) \\
& =\int d p^{0} \frac{1}{\left[\left(p^{0}+i \epsilon\right)^{2}-p^{2}\right]\left[\left(p^{0}+k-i \epsilon\right)^{2}-|\mathbf{p}+\mathbf{k}|^{2}\right]} \\
& \sim \frac{1}{T^{2}} \times \frac{1}{p+k-|\mathbf{p}+\mathbf{k}|} \sim \frac{1}{g^{2} T^{3}}
\end{aligned}
$$

where we did a contour integration and used that $\hat{\mathbf{p}} \cdot \hat{\mathbf{k}}=$ $1-\mathcal{O}\left(g^{2}\right)$. In real calculations one must use resummed fermion propagators since their self-energy is $\mathcal{O}\left(g^{2}\right)$. Finally each gluon vertex contributes a factor $g$ and each photon vertex contributes a factor $e$ as well as a factor $g$ because of kinematics [20. Including a $g^{3}$ phase space suppression because $\mathbf{q}$ is soft and a $g^{2}$ suppression because $\mathbf{p}$ is collinear with $\mathbf{k}$ one sees that the diagram is of order $g^{2} e^{2}$. A similar analysis shows that Fig. 4 is also leading order.

The above argument relied mostly on kinematics and is therefore equally valid in non-equilibrium systems. ${ }^{1}$ Nevertheless, it assumed thermal equilibrium in two crucial places. Firstly, the authors of 20] used a KMS condition for four-point functions to show that only $S_{r a}$ and $S_{a r}$ contribute to the pinching poles. We provide a more general argument in the next two sections. Secondly, Eq. (27) for the $r r$ propagator was derived using the KMS condition.

In general the retarded self-energy for soft gluons is [23, 35]

$$
\begin{aligned}
\Pi_{\mathrm{ret}}^{\mu \nu}(Q)= & -2 g^{2} \int \frac{d^{3} p}{(2 \pi)^{3}} \frac{1}{2 p}\left(\frac{\partial f_{\mathrm{tot}}(\mathbf{p})}{\partial P^{\omega}}\right) \\
& \times\left[-P^{\mu} g^{\omega \nu}+\frac{Q^{\omega} P^{\mu} P^{\nu}}{P \cdot Q+i \epsilon}\right]
\end{aligned}
$$

where

$$
f_{\text {tot }}=N_{f} f_{q}+N_{f} f_{\bar{q}}+2 N_{c} f_{g}
$$

with $f_{q}, f_{\bar{q}}, f_{g}$ the distribution for quarks, antiquarks and gluons respectively. We should interpret $\partial f_{\text {tot }}(\mathbf{p}) / \partial p^{0}=$ 0 . The 12 component, $\Pi_{<}(Q)$, has also been evaluated for space-like gluons [23]. It is

$$
\begin{aligned}
\Pi_{<}^{\mu \nu}(Q)=-\left.i g^{2} \int \frac{d^{3} p}{(2 \pi)^{3}} \frac{P^{\mu} P^{\nu}}{p} 2 \pi \delta(P \cdot Q)\right|_{p^{0}=p} \\
\times\left[N_{f} f_{q}(\mathbf{p})\left(1-f_{q}(\mathbf{p})\right)+N_{f} f_{\bar{q}}(\mathbf{p})\left(1-f_{\bar{q}}(\mathbf{p})\right)\right. \\
\left.+2 N_{c} f_{g}(\mathbf{p})\left(1+f_{g}(\mathbf{p})\right)\right] .
\end{aligned}
$$

\footnotetext{
${ }^{1}$ In systems that are far away from thermal equilibrium there is no well defined temperature. Then the scale $T$ should be replaced by the hard scale which contributes to the greatest number of particles, see [23] for further details.
}

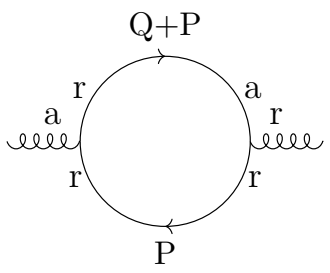

FIG. 5. One of the diagrams contributing to $\Pi_{\mathrm{ret}}=\Pi_{a r}$

In both expressions we have used that $P \gg Q$. Eq. (31) has an intuitive interpretation. The soft gluons are sourced by hard quarks and gluons with momentum P. The factor $f_{q}(\mathbf{p})\left(1-f_{q}(\mathbf{p})\right)$ is the density of the incoming and outgoing hard quark including Pauli blocking. Similarly $f_{g}(\mathbf{p})\left(1+f_{g}(\mathbf{p})\right)$ describes the hard gluon. These expressions are true as long as the hard thermal loop (HTL) scheme is valid. This puts some mild constraints on the momentum distribution [23, such as that the density of soft gluons cannot be exceedingly high.

We can now see that $G_{r r} \sim g^{-3}$ for soft gluons in nonequilibrium systems. Clearly $\Pi_{\text {ret }} \sim g^{2} T^{2}$ so $G_{\text {ret }} \sim g^{-2}$ while $\Pi_{<} \sim g T^{2}$ because of the delta function in Eq. (31). Thus, using Eq. 20,

$$
G_{r r} \approx G_{\mathrm{ret}}\left(-i \Pi_{<}\right) G_{\mathrm{adv}} \sim \frac{1}{g^{3}}
$$

which ensures that the LPM effect matters at leading order.

At first sight it might be surprising that $\Pi_{<}$and $\Pi_{\text {ret }}$ differ by a power of $g$ since they come from the same Feynman diagrams. The reason for the difference is the following: The vertices give factors $P^{\mu} P^{\nu}$ which are $\mathcal{O}(1)$ and factors $P^{\mu} Q^{\nu}+Q^{\mu} P^{\nu}$ and $P \cdot Q g^{\mu \nu}$ which are $\mathcal{O}(g)$. For the retarded self-energy all terms with $P^{\mu} P^{\nu}$ cancel giving a suppression in $g$. As an example we can look at Fig. 5 The diagram goes like

$$
\begin{aligned}
& \int d^{4} P \frac{P^{\mu} P^{\nu} \delta\left(P^{2}\right)}{(Q+P)^{2}+i \epsilon\left(q^{0}+p^{0}\right)} \\
& \left.\sim \int d^{3} p \frac{P^{\mu} P^{\nu}}{2 q^{0} p^{0}-2 \mathbf{p} \cdot \mathbf{q}+i \epsilon p^{0}}\right|_{p^{0}=p} \\
& +\left.\int d^{3} p \frac{P^{\mu} P^{\nu}}{2 q^{0} p^{0}-2 \mathbf{p} \cdot \mathbf{q}+i \epsilon p^{0}}\right|_{p^{0}=-p}
\end{aligned}
$$

at leading order. The two terms cancel as can be seen by doing $\mathbf{p} \rightarrow-\mathbf{p}$ in the last integral. Such a cancellation does not take place when the vertex factor is $P^{\mu} Q^{\nu}+$ $Q^{\mu} P^{\nu}$ or $P \cdot Q g^{\mu \nu}$.

The gluon $r r$ propagator is more complicated in a nonequilibrium plasma than in equilibrium. In particular it has an imaginary part. Using the definition

$$
G_{r r}^{\mu \nu}(P)=\frac{1}{2} \int d^{4} x e^{i P \cdot x}\left\langle\left\{A^{\mu}(x), A^{\nu}(0)\right\}\right\rangle
$$


we get that $G_{r r}^{\mu \nu}(P)^{*}=G_{r r}^{\mu \nu}(-P)$. In translationally invariant systems we furthermore get that

$$
\begin{aligned}
G_{r r}^{\mu \nu}(P) & =\frac{1}{2} \int d^{4} x e^{-i P \cdot x}\left\langle\left\{A^{\mu}(-x), A^{\nu}(0)\right\}\right\rangle \\
& =\frac{1}{2} \int d^{4} x e^{-i P \cdot x}\left\langle\left\{A^{\nu}(x), A^{\mu}(0)\right\}\right\rangle \\
& =G_{r r}^{\nu \mu}(-P)
\end{aligned}
$$

where we did a change of variables $x \rightarrow-x$ in the first line and translated the propagator in the second line. These results are clearer when writing

$$
G_{r r}^{\mu \nu}(P)=\operatorname{Re} G_{r r}^{\mu \nu}(P)+i \operatorname{Im} G_{r r}^{\mu \nu}(P) .
$$

Then $\operatorname{Re} G_{r r}^{\mu \nu}(-P)=\operatorname{Re} G_{r r}^{\mu \nu}(P)$ and $\operatorname{Im} G_{r r}^{\mu \nu}(-P)=$ $-\operatorname{Im} G_{r r}^{\mu \nu}(P)$. Furthermore, Eq. (35) shows that $\operatorname{Re} G_{r r}$ is symmetric and $\operatorname{Im} G_{r r}$ is antisymmetric under the interchange of the spacetime indices. Evaluation of $G_{r r}$ explicitly given some momentum distribution function $f$ is a subject for future research. The 00 component has been evaluated using an anisotropic momentum distribution as it has applications to the heavy-quark potential in QGP [36].

The imaginary part of $G_{r r}$ might seem surprising. It is helpful to consider how it comes about. We can always write

$$
G_{r r}=G_{\mathrm{ret}} \frac{-i\left(\Pi_{>}+\Pi_{<}\right)}{2} G_{\mathrm{adv}} .
$$

where

$$
\left(1-G_{\text {ret }}^{0}\left(-i \Pi_{\text {ret }}\right)\right) G_{\text {ret }}=G_{\text {ret }}^{0}
$$

and similarly for $G_{\mathrm{adv}}$. The bare propagator and the HTL self energy is symmetric in the spacetime indices so the same goes for $G_{\text {ret }}$ and $G_{\text {adv }}$. Thus $G_{r r}$ is the product of three symmetric matrices. In general

$$
G_{r r}^{T}=G_{\mathrm{adv}} \frac{-i\left(\Pi_{>}+\Pi_{<}\right)}{2} G_{\mathrm{ret}}
$$

will be different from $G_{r r}$ because these matrices do not commute. In equilibrium (and for any isotropic momentum distribution) the only available tensors are $g^{\mu \nu}$, the external momentum $P^{\mu}$ and the plasma four-velocity, $u^{\mu}$. Therefore all matrices are spanned by $g^{\mu \nu}, P^{\mu} P^{\nu}$ and the projection operators $P_{T}$ and $P_{L}$ [37]. These four matrices commute so $G_{r r}^{T}=G_{r r}$ and $G_{r r}$ is real. In an anisotropic plasma there are additional tensors describing the anisotropy and therefore more matrices. They will not all commute in general giving $G_{r r}$ an imaginary part.

\section{THE OCCUPATION DENSITY OF HARD QUARKS}

To evaluate the LPM effect we need the $r r$ propagator for hard and nearly on-shell quarks, i.e. $S_{r r}(P)$ with

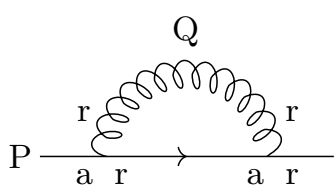

(a)

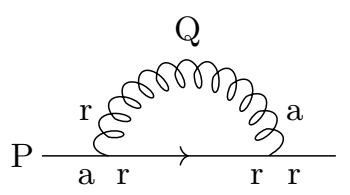

(b)

FIG. 6. Diagrams contributing to $\Sigma_{\text {ret }}$ at leading order in $g$.

$P \sim T$ and $P^{2} \sim g^{2} T^{2}$. We will show that at leading order

$$
S_{r r}=\left[\frac{1}{2}-F\right]\left(S_{\mathrm{ret}}-S_{\mathrm{adv}}\right),
$$

just as for scalars. Here $F(P):=-P \cdot \Sigma_{<} / 2 i P \cdot \operatorname{Im} \Sigma_{\text {ret }}$ is a resummed occupation density.

We begin by evaluating $\Sigma_{\text {ret }}$. The contributing diagrams can be seen in Fig. 6. For an internal particle with soft momentum, $\mathcal{O}(g T)$, we must use a HTL resummed propagator, while for hard particles we use bare propagators. There are a few different momentum regimes. When the loop momentum is hard, $Q \sim T$, the two diagrams give rise to the thermal mass

$$
\begin{aligned}
m_{\infty}^{2} & =2 P \cdot \operatorname{Re} \Sigma_{\text {ret }}(P) \\
& =2 g^{2} C_{F} \int \frac{d^{3} p}{(2 \pi)^{3}} \frac{2 f_{g}(\mathbf{p})+f_{q}(\mathbf{p})+f_{\bar{q}}(\mathbf{p})}{2 p} .
\end{aligned}
$$

The contribution of this momentum regime to $\operatorname{Im} \Sigma_{\text {ret }}$ is phase space suppressed because both $G_{r r}^{0}$ and $\operatorname{Im} S_{\text {ret }}^{0}$ contain a delta function forcing the internal particles to be on shell 2

We now focus on the top diagram in Fig. 6 which is given by

$$
\left.\Sigma_{\mathrm{ret}}(P)\right|_{(a)}=-i g^{2} C_{F} \int \frac{d^{4} Q}{(2 \pi)^{4}} G_{r r}^{\mu \nu}(Q) \gamma_{\mu} S_{\mathrm{ret}}(P-Q) \gamma_{\nu} .
$$

When the gluon is soft and the quark is hard the leading order contribution is

$$
\begin{aligned}
\left.\Sigma_{\text {ret }}(P)\right|_{\text {soft }} & =g^{2} C_{F} \int \frac{d^{4} Q}{(2 \pi)^{4}} \gamma_{\mu} \not P \gamma_{\nu} G_{r r}^{\mu \nu}(Q) \\
\times & {\left[-i \pi \operatorname{sgn}\left(p^{0}\right) \delta(2 P \cdot Q)+\frac{1}{-2 P \cdot Q}\right] }
\end{aligned}
$$

where we have substituted the bare quark propagator. We have used that the quark is on-shell, $P^{2} \sim g^{2} T^{2}$.

\footnotetext{
2 In our notation $\Sigma_{\text {ret }}$ can denote both a spinor matrix and a fourvector, i.e. $\Sigma_{\text {ret }}=\Sigma_{\text {ret }}^{\mu} \gamma_{\mu}$.
} 
Using the properties of $G_{r r}^{\mu \nu}(Q)$ under the interchange of the spacetime indices and under $Q \rightarrow-Q$ we can write

$$
\left.\Sigma_{\mathrm{ret}}(P)\right|_{\mathrm{soft}}=\operatorname{Re} \Sigma_{\mu}^{\mathrm{p}} \gamma^{5} \gamma^{\mu}+i \operatorname{Im} \Sigma_{\mu} \gamma^{\mu}
$$

where

$$
\begin{gathered}
\operatorname{Im} \Sigma_{\omega}=-g^{2} C_{F} \int^{g T} \frac{d^{4} Q}{(2 \pi)^{4}}\left(P_{\mu} g_{\nu \omega}+P_{\nu} g_{\mu \omega}-g_{\mu \nu} P_{\omega}\right) \\
\times \pi \operatorname{sgn}\left(p^{0}\right) \delta(2 P \cdot Q) \operatorname{Re} G_{r r}^{\mu \nu}
\end{gathered}
$$

and we have a pseudovector component

$$
\operatorname{Re} \Sigma_{\omega}^{\mathrm{p}}=g^{2} C_{F} \varepsilon_{\omega \rho \mu \nu} P^{\rho} \int^{g T} \frac{d^{4} Q}{(2 \pi)^{4}} \frac{\operatorname{Im} G_{r r}^{\mu \nu}}{2 P \cdot Q} .
$$

Here we used the identity 38

$$
\gamma_{\mu} \gamma_{\rho} \gamma_{\nu}=g_{\mu \rho} \gamma_{\nu}+g_{\rho \nu} \gamma_{\mu}-g_{\mu \nu} \gamma_{\rho}+i \epsilon_{\sigma \mu \rho \nu} \gamma^{\sigma} \gamma^{5}
$$

to separate the symmetrical and antisymmetrical part of $G_{r r}$. The expression in Eq. (44) is clearly leading order because $G_{r r} \sim g^{-3} T^{2}$ and $Q \sim g T$. The vector term with $\operatorname{Im} \Sigma_{\mu}$ is both present in equilibrium and non-equilibrium plasma. It determines the decay width of hard quarks, $\Gamma$, through

$$
\begin{aligned}
-\frac{1}{2} p^{0} \Gamma & =P \cdot \operatorname{Im} \Sigma_{\text {ret }} \\
& =-2 \pi g^{2} C_{F} \operatorname{sgn}\left(p^{0}\right) \\
& \times P_{\mu} P_{\nu} \int^{g T} \frac{d^{4} Q}{(2 \pi)^{4}} \operatorname{Re} G_{r r}^{\mu \nu}(Q) \delta(2 P \cdot Q) .
\end{aligned}
$$

The pseudovector term with $\operatorname{Re} \Sigma_{\mu}^{p}$ is only present in anisotropic systems because it includes the imaginary part of $G_{r r}$.

There are no further leading order contributions to $\Sigma_{\text {ret }}$. The case of a soft internal quark, $P-Q \sim g T$, in part (a) of Fig. 6 is subleading because there is no enhancement from soft gluons. Similarly, diagram (b) of the same figure is subleading when either particle is soft because the $g^{-3}$ contribution from $G_{r r}$ is missing.

We can now derive the retarded quark propagator. We have shown that the full self-energy is

$$
\Sigma_{\text {ret }}=\Sigma_{\mu} \gamma^{\mu}+\operatorname{Re} \Sigma_{\mu}^{\mathrm{p}} \gamma^{5} \gamma^{\mu}
$$

where $\operatorname{Re} \Sigma_{\mu}$ gives the thermal mass in Eq. (41) and $\operatorname{Im} \Sigma_{\mu}$ and $\operatorname{Re} \Sigma_{\mu}^{\mathrm{p}}$ are as before. The retarded propagator in the Dirac representation is then

$$
S_{\text {ret }}=\left[\begin{array}{cc}
0 & \frac{i\left(P-\Sigma+\operatorname{Re} \Sigma^{\mathrm{p}}\right) \cdot \sigma}{\left(P-\Sigma+\operatorname{Re} \Sigma^{\mathrm{p}}\right)^{2}} \\
\frac{i\left(P-\Sigma-\operatorname{Re} \Sigma^{\mathrm{p}}\right) \cdot \bar{\sigma}}{\left(P-\Sigma-\operatorname{Re} \Sigma^{\mathrm{p}}\right)^{2}} & 0
\end{array}\right]
$$

Because of the $\gamma^{5}$ matrix the pseudovector part of the self-energy, $\operatorname{Re} \Sigma^{\mathrm{p}}$, has different signs for positive and negative helicities. However, both helicities have the same thermal mass since $P \cdot \operatorname{Re} \Sigma_{\text {ret }}^{p}$ vanishes because of the Levi-Civita tensor. The imaginary part of the self energy still has the same sign for both helicities. Thus we can write

$$
S_{\text {ret }}=\frac{i \not p}{P^{2}-m_{\infty}^{2}+i \Gamma p^{0}} .
$$

at leading order where $m_{\infty}^{2}$ is given by Eq. 411. We have used that $(P-\Sigma)^{2} \approx P^{2}-2 P \cdot \Sigma$. We see that the pseudovector component, and therefore $\operatorname{Im} G_{r r}$, does not contribute when considering on-shell particles which simplifies the calculations considerably.

We can finally derive $S_{r r}$ in Eq. 40. A similar argument as for $\Sigma_{\text {ret }}$ shows that

$$
\begin{aligned}
P \cdot \Sigma_{<}(P)= & 4 \pi i g^{2} C_{F}\left[f_{q}(\mathbf{p}) \theta\left(p^{0}\right)+\left(f_{\bar{q}}(-\mathbf{p})-1\right) \theta\left(-p^{0}\right)\right] \\
& \times P_{\mu} P_{\nu} \int^{g T} \frac{d^{4} Q}{(2 \pi)^{4}} \operatorname{Re} G_{r r}^{\mu \nu}(Q) \delta(2 P \cdot Q)
\end{aligned}
$$

The quark momentum distribution comes from $S_{12}^{0}$ and we have used that $G_{12} \approx G_{r r}$ for soft gluons. Since

$$
\not P \dot{\psi}_{<} \not P=-P^{2} \dot{\psi}_{<}+2 P \cdot \Sigma_{<} \not P \approx 2 P \cdot \Sigma_{<} \not P
$$

one can easily show that at leading order

$$
\begin{aligned}
S_{r r}(P) & =\frac{1}{2}\left(S_{\mathrm{ret}}-S_{\mathrm{adv}}\right)+S_{\mathrm{ret}}\left(-i \Sigma_{<}\right) S_{\mathrm{adv}} \\
& \approx\left(\frac{1}{2}-F(P)\right)\left(S_{\mathrm{ret}}-S_{\mathrm{adv}}\right) .
\end{aligned}
$$

where

$F(P):=-\frac{P \cdot \Sigma_{<}}{2 i P \cdot \operatorname{Im} \Sigma_{\text {ret }}}=f_{q}(\mathbf{p}) \theta\left(p^{0}\right)+\left(1-f_{\bar{q}}(-\mathbf{p})\right) \theta\left(-p^{0}\right)$.

All dependence on $G_{r r}$ cancels out in $F$.

Comparing with the expression for $S_{r r}$ in equilibrium we see that $F$ should be interpreted as a resummed occupation density. Its form makes perfect sense. In the Boltzmann equation incoming particles have $p^{0}>0$ and outgoing particles have $p^{0}<0$. Thus $F$ is just the bare momentum distribution with Pauli blocking for outgoing quarks. This function reduces to the Fermi-Dirac distribution $f_{F}\left(p^{0}\right)$ in equilbrium as can be seen by using $1-f_{F}(-x)=f_{F}(x)$ and noting that when going from equilibrium to non-equilibrium systems one makes the identification $f_{F}\left(\left|p^{0}\right|\right) \leftrightarrow f_{q}(\mathbf{p})$.

\section{THE LPM EFFECT IN A NON-EQUILIBRIUM PLASMA}

We now have all the ingredients to evaluate the LPM effect in an out-of-equilibrium quark-gluon plasma, and to compute the photon production rate in Eq. 26]. The photon sources are connected to a quark and an antiquark so we need to evaluate the four-point function

$$
S_{1122}\left(x_{1}, x_{2} ; y_{1}, y_{2}\right)=\left\langle T_{\mathcal{C}}\left\{\bar{\psi}_{1}\left(x_{1}\right) \psi_{1}\left(x_{2}\right) \bar{\psi}_{2}\left(y_{1}\right) \psi_{2}\left(y_{2}\right)\right\}\right\rangle \text {. }
$$




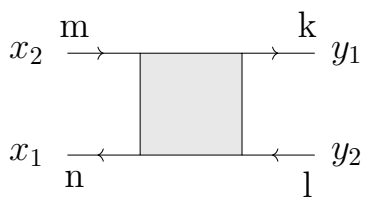

(a)

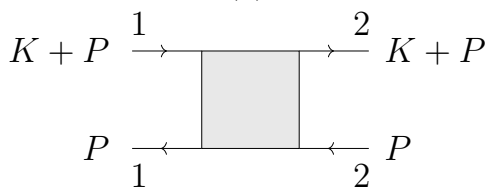

(b)

FIG. 7. Definition of the four-point function $S_{n m k l}\left(x_{1}, x_{2} ; y_{1}, y_{2}\right)$ in position space. $n, m, k, l$ are either 1 or 2 . Also shown is the diagram we need to evaluate, i.e. $S_{1122}$ in momentum space.

See Fig. 7, top diagram, for the corresponding contribution. When going to momentum space we can approximate the momentum in the quark or the antiquark rail as constant because it only changes through the exchange of soft gluons. The relevant diagram is the bottom one in Fig. 7 where $K$ is the photon momentum and $P$ is the loop momentum.

\section{A. Summing four-point functions without the KMS condition}

Up until now our analysis has been in the $r / a$ basis which enables power counting of the complicated diagrams. We must evaluate $S_{1122}$ using the expression

$$
\begin{aligned}
& S_{1122}=S_{r r r r}+\frac{1}{2}\left(S_{a r r r}+S_{r a r r}-S_{\text {rrar }}-S_{\text {rrra }}\right) \\
& \quad+\frac{1}{4}\left(S_{a a r r}-S_{\text {arar }}-S_{a r r a}-S_{\text {raar }}-S_{\text {rara }}+S_{\text {rraa }}\right) \\
& \quad+\frac{1}{8}\left(S_{\text {raaa }}+S_{\text {araa }}-S_{\text {aara }}-S_{\text {aaar }}+\frac{1}{2} S_{a a a a}\right) .
\end{aligned}
$$

This task might look overwhelming. Each four-point function on the right hand side is a sum of infinitely many diagrams with a different number of gluon rungs. They need not have any clear pattern in their $r / a$ indices.

In thermal equilibrium the four-point functions have been related using the KMS condition [39]. Specifically,

$$
\begin{aligned}
S_{1122}= & \alpha_{1} S_{\text {aarr }}+\alpha_{2} S_{\text {aaar }}+\alpha_{3} S_{\text {aara }}+\alpha_{4} S_{\text {araa }} \\
& +\alpha_{5} S_{\text {raaa }}+\alpha_{6} S_{\text {arra }}+\alpha_{7} S_{\text {arar }}+\text { c.c. }
\end{aligned}
$$

where the coefficients depend on the Fermi-Dirac distribution. As an example $\alpha_{1}=f_{F}\left(p^{0}+k^{0}\right)\left(1-f_{F}\left(p^{0}\right)\right)$. One can also show that $S_{\text {aarr }}$ is the only one of these four-point functions that contributes at leading order. Therefore

$$
S_{1122}=2 f_{F}\left(p^{0}+k^{0}\right)\left(1-f_{F}\left(p^{0}\right)\right) \operatorname{Re} S_{a a r r}
$$

in thermal equilibrium.

We will now evaluate $S_{1122}$ generally without using the KMS condition. Our derivation is thus also valid in nonequilibrium systems. It only relies on the power counting scheme. We know that all gluon rungs must be $r r$ propagators to get the $1 / \mathrm{g}$ enhancement from the density of soft gluons. Each vertex contains an odd number of $a$ indices so one quark propagator ends with $a$ and one with $r$ at each vertex, see Fig. 9. Finally $a a$ propagators vanish.

We first consider quark rails as in Fig. 8. For the convenience of the reader we draw $S_{a r}$ with red, dashed lines, $S_{r a}$ with blue, dotted lines and $S_{r r}$ with black lines. The first propagator in a quark rail that starts with $a$ must be $S_{a r}$. The next propagator must then start with $a$ and so on. Thus all propagators are $S_{a r}$ at leading order. Similarly all propagators in a quark rail that starts with $r$ and ends with $a$ are $S_{r a}$. Finally, quark rails that start and end with $a$ vanish. This means that we can ignore $S_{\text {araa }}, S_{\text {aara }}, S_{\text {arra }}, S_{\text {raar }}, S_{\text {aaar }}, S_{\text {raaa }}$ and $S_{\text {aaaa }}$ at leading order. (In fact, $S_{a a a a}$ vanishes at all orders [39.)

There are more possibilities for quark rails that start and end with $r$. It is easy to see that they consist of arbitrarily many $S_{r a}$, then one $S_{r r}$, and finally arbitrarily many $S_{a r}$. Thus there are $n$ possibilities for a quark rail with $n$ propagators which differ in the placement of $S_{r r}$. See Fig. 8 for the case of three propagators.

The remaining four-point functions can only contribute at leading order if we get pinching poles from each pair of propagators between adjacent gluon rungs. Just like in Eq. 228 that means that one propagator is $S_{r a}$ and the other one $S_{a r}$, see Fig. 10 This immediately tells us that $S_{\text {rara }}$ and $S_{\text {arar }}$ can be discarded because they have no pinching poles, see Fig. 11

We can now express the remaining seven four-point functions in terms of $S_{a a r r}$ and $S_{\text {rraa }}$. They all include an $r r$ propagator the pole structure of which can be seen from

$$
S_{r r}=\left[\frac{1}{2}-F(P)\right]\left(S_{r a}-S_{a r}\right) .
$$

It has poles on both sides of the real axis. At leading order we can drop the term that does not give a pinching pole. As an example we must place the indices in $S_{\text {rarr }}$ as in Fig. 12 to get pinching poles from all pairs. Then $S_{r r}$ is on the far left and only the term with $S_{r a}$ contributes. Thus

$$
S_{\text {rarr }}=\left(\frac{1}{2}-F(P)\right) S_{a a r r}
$$

where the leading order diagram for $S_{\text {aarr }}$ is in Fig. 13 . 


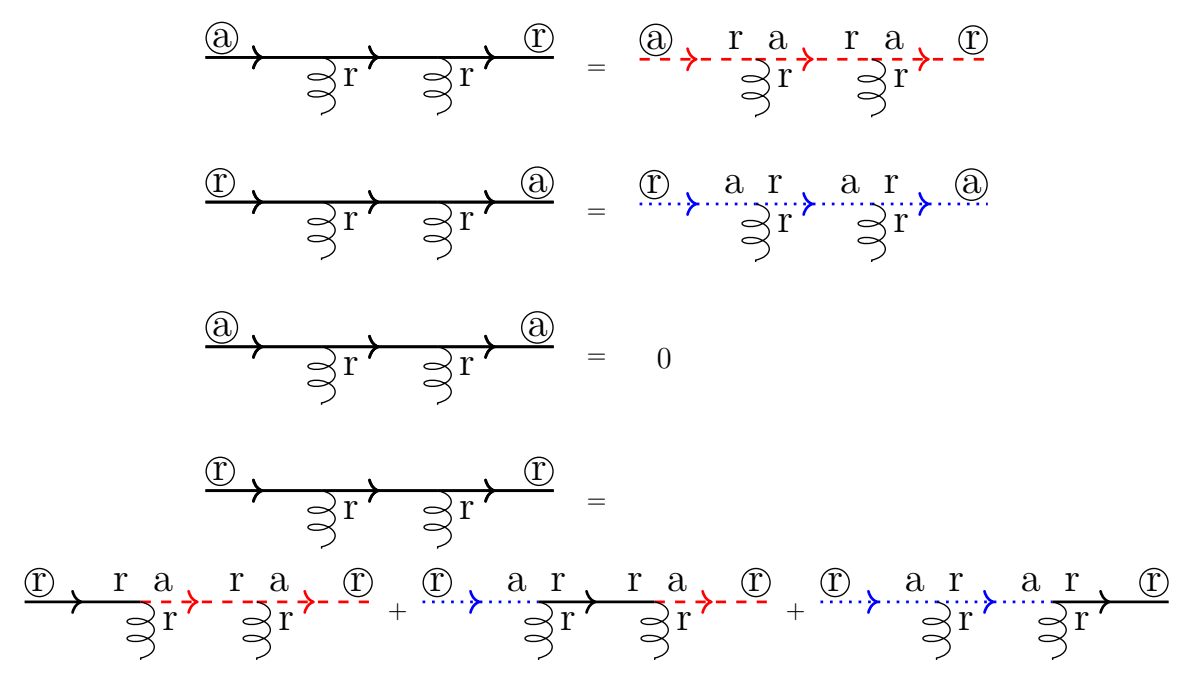

FIG. 8. (Color online) Analysis of quark rails in the $r / a$ basis.

$$
\frac{r a}{2 r} \frac{a r}{2 r}
$$

FIG. 9. (Color online) Vertices that contribute at leading order.

$$
\begin{aligned}
& \mathrm{P}+\mathrm{K}-\stackrel{\mathrm{a}}{-}-\rightarrow-\mathrm{r} . \quad \mathrm{P}+\mathrm{K} \stackrel{\mathrm{r}}{\cdots \cdots} \rightarrow \cdots \cdots
\end{aligned}
$$

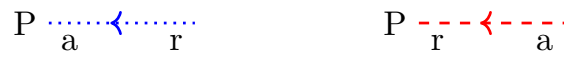

FIG. 10. (Color online) The pairs of propagators that give pinching poles.

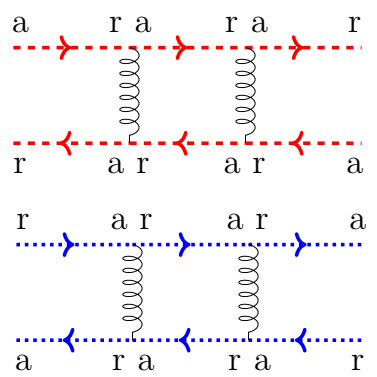

FIG. 11. (Color online) $S_{\text {rara }}$ and $S_{\text {arar. }}$ These diagrams do not contribute at leading order because there are no pinching poles.

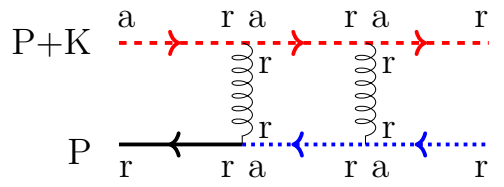

FIG. 12. (Color online) The only way of placing $r / a$ indices in $S_{\text {rarr }}$ at leading order. In a general diagram with arbitrarily many gluon rungs $S_{r r}$ must still be on the far left.

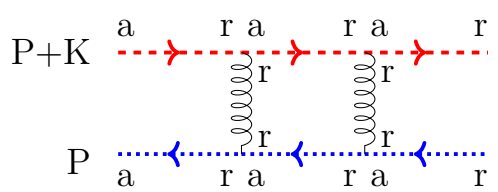

FIG. 13. (Color online) The only way of placing $r / a$ indices in $S_{\text {aarr }}$ at leading order.

Similarly, one sees that

$$
\begin{aligned}
& S_{\text {rrar }}=-\left(\frac{1}{2}-F(P)\right) S_{\text {rraa }} \\
& S_{a r r r}=-\left(\frac{1}{2}-F(P+K)\right) S_{a a r r} \\
& S_{\text {rrra }}=\left(\frac{1}{2}-F(P+K)\right) S_{\text {rraa }} .
\end{aligned}
$$

To finish our derivation, we analyze $S_{r r r}$ which is more complicated. Each quark rail has one $r r$ propagator. The two $S_{r r}$ must be on top of each other or immediately diagonal to each other since otherwise we will miss a pinching pole pair, see Fig. 14. Let's consider the case of two gluon rungs. In Fig. 15 we analyze the three possibilities of having the $r r$ on top of each other. The remaining possibilities are analyzed in Fig. 16. When these contributions are summed over, all terms cancel except for

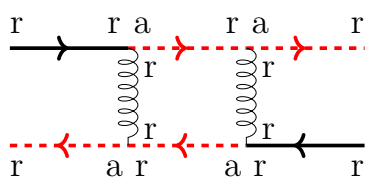

FIG. 14. (Color online) An example of a diagram that does not contribute to $S_{\text {rrrr }}$ at leading order because the propagators in the middle do not give pinching poles. 


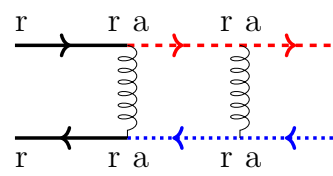

$$
=-\left(\frac{1}{2}-F(P)\right)\left(\frac{1}{2}-F(P+K)\right)
$$
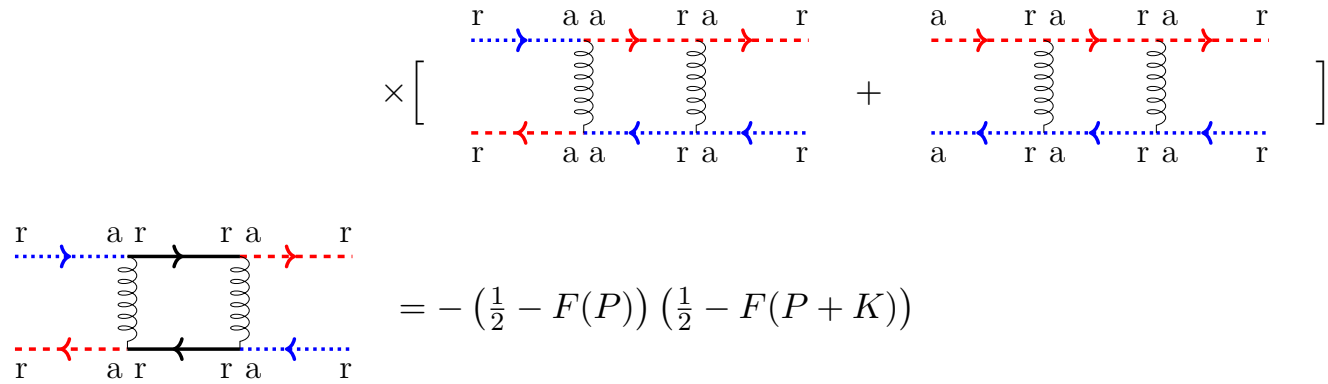

$=-\left(\frac{1}{2}-F(P)\right)\left(\frac{1}{2}-F(P+K)\right)$
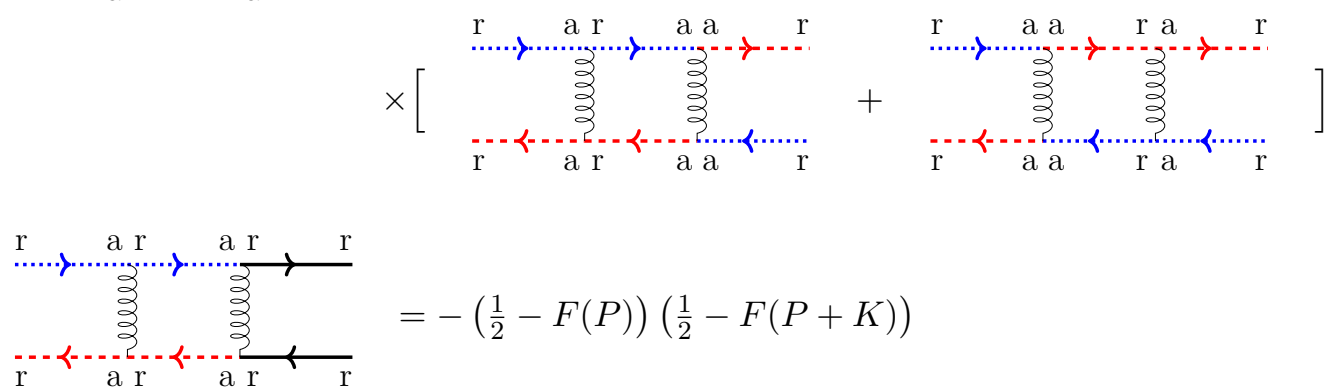

$=-\left(\frac{1}{2}-F(P)\right)\left(\frac{1}{2}-F(P+K)\right)$

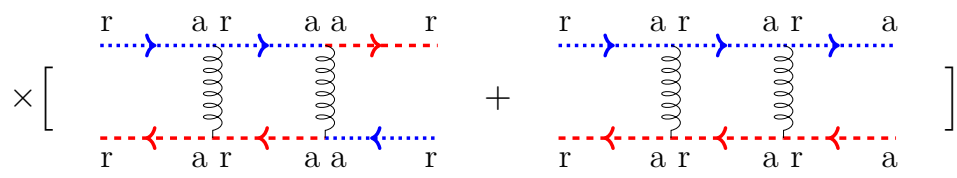

FIG. 15. (Color online) Leading order contributions to $S_{r r r r}$ where the $S_{r r}$ propagators are on top of each other.

those corresponding to $S_{a a r r}$ and $S_{\text {rraa }}$, see Fig. 17. A similar cancellation takes place for any number of gluon rungs. Specifically, four-point functions with $S_{r r}$ immediately diagonal to each other cancel out with four-point functions with $S_{r r}$ on top of each other. We are then left with

$S_{r r r r}=-\left(\frac{1}{2}-F(P)\right)\left(\frac{1}{2}-F(P+K)\right)\left[S_{r r a a}+S_{a a r r}\right]$.

We have evaluated all terms in Eq. (57) at leading order. Summing up Eq. (61) to 65) we get that

$$
S_{1122}=F(P+K)(1-F(P))\left[S_{\text {rraa }}+S_{\text {aarr }}\right] .
$$

This can be rewritten using $S_{\text {rraa }}=S_{\text {aarr }}^{*}$ which can be seen from the definition of the four-point functions or from $S_{r a}^{*}=-S_{a r}$. We have thus shown that

$$
S_{1122}=2 F(P+K)(1-F(P)) \operatorname{Re} S_{a a r r}
$$

without using the KMS condition. This expression is convenient because $S_{\text {aarr }}$ has a very simple structure, see Fig. 18. In thermal equilibrium $F$ from Eq. (55) reduces to the Fermi-Dirac distribution and we retrieve the equilibrium result, Eq. (59).
The factors of $F$ in Eq. (67) give the momentum distribution of incoming and outgoing quarks including Pauli blocking. Our analysis is valid for on-shell photons, $k^{0} \approx k$. The momentum regime where $p^{0}>0$ represents bremsstrahlung off a quark with initial momentum $\mathbf{k}+\mathbf{p}$. The distribution functions are $f_{q}(\mathbf{k}+\mathbf{p})\left(1-f_{q}(\mathbf{p})\right)$ as expected because there is one incoming and one outgoing quark. Bremsstrahlung off an antiquark is given by $p^{0}<-k$. The antiquark's initial momentum in the photon's direction is $-p^{0}$ and the final momentum is $-\left(k+p^{0}\right)$. The distribution functions are then $\left(1-f_{\bar{q}}(-\mathbf{k}-\mathbf{p})\right) f_{\bar{q}}(-\mathbf{p})$. The antiquark distributions are evaluated at negative momentum because we defined the momentum to flow in the direction of quarks. Finally the momentum regime $-k<p^{0}<0$ corresponds to the pair annihilation of a quark with momentum $k+p^{0}$ and an antiquark with momentum $-p^{0}$. The distribution functions are $f_{q}(\mathbf{k}+\mathbf{p}) f_{\bar{q}}(-\mathbf{p})$.

\section{B. Summing ladder diagrams}

The only remaining task is to sum up the ladder diagrams contributing to $S_{a a r r}$. This gives an integral equa- 

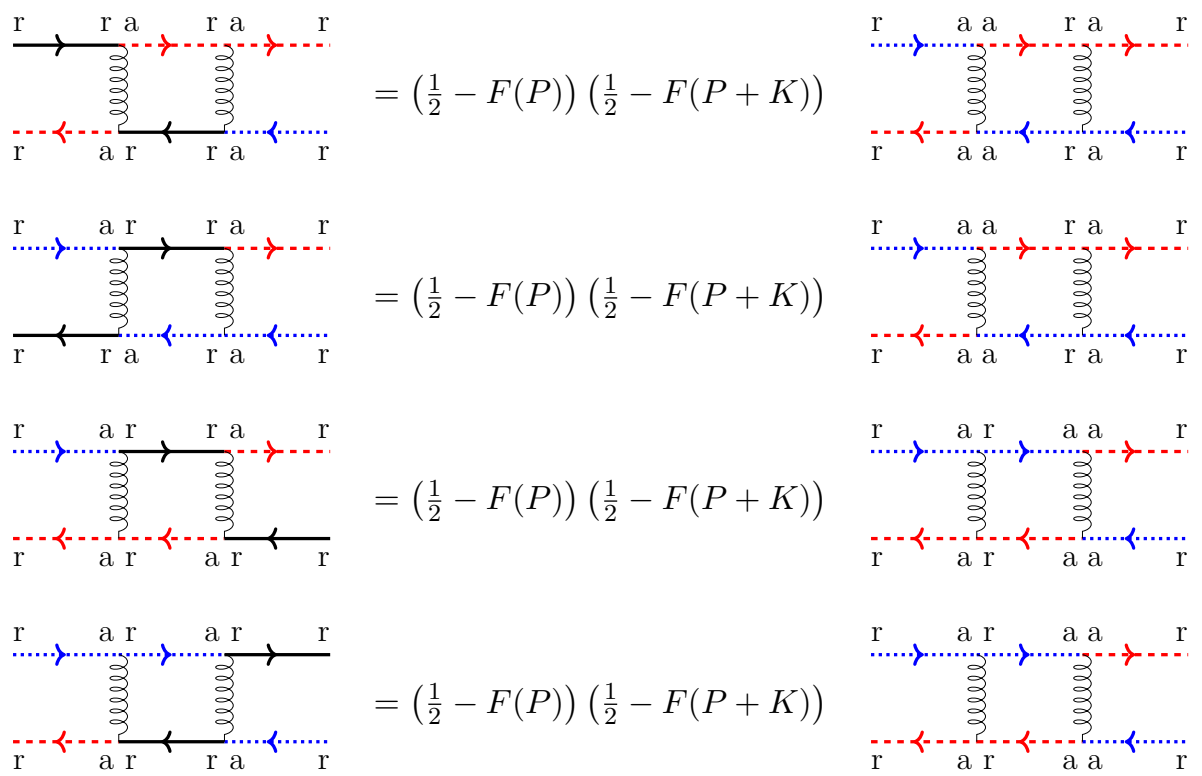

FIG. 16. (Color online) Leading order contributions to $S_{r r r r}$ where the $S_{r r}$ propagators are immediately diagonal to each other.

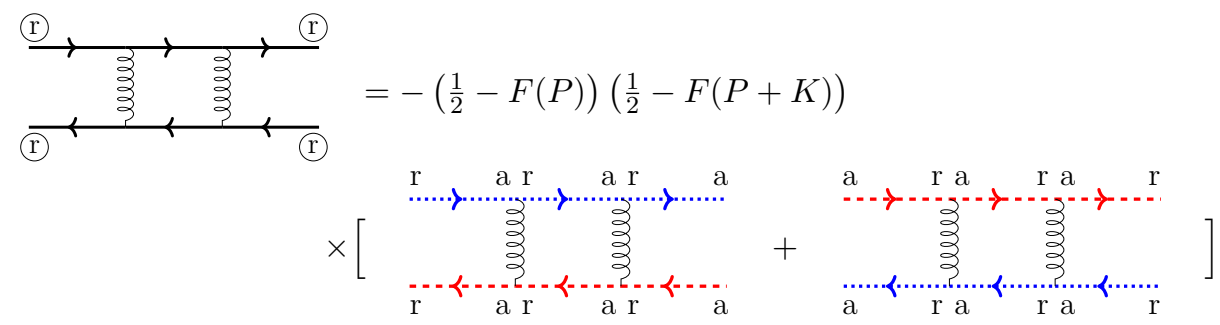

FIG. 17. (Color online) All leading order contributions to $S_{r r r r}$ after cancellation.

tion which describes the LPM effect. We will outline the derivation briefly as it is quite similar to the one in thermal equilibrium, see 20, 40, for further details. The notation follows that of Ref. 41. The contributing diagrams are shown in Fig. 19 and the procedure for summing them up is in Fig. 20 and 21. One gets an integral equation for the resummed vertex $\mathcal{D}(P, P+K)$ which can be written schematically as

$$
\mathcal{D}^{\mu}=\mathcal{I}^{\mu}+\int \frac{d^{4} Q}{(2 \pi)^{4}} \mathcal{M F} \mathcal{D}^{\mu}
$$

Here $\mathcal{I}^{\mu}$ is the bare vertex, $\mathcal{F}(P, P+K)$ are quark propagators that give pinching poles and $\mathcal{M}(P, P+K)$ is the contribution from a gluon ladder. The gluon momentum $Q$ is soft, $K$ is the photon momentum and $P$ is the momentum in one of the quark rails. We need to evaluate $\mathcal{F}$ and $\mathcal{M}$.

We let the photon propagate in the z-direction. The collinear momentum of the quark momentum is hard, $p^{z} \sim T$, but the orthogonal components are soft, $\mathbf{p}_{\perp} \sim$ $g T$. Furthermore the quark is nearly on shell, $p^{0}=$ $p^{z}+\mathcal{O}\left(g^{2} T\right)$. It is convenient to decompose the quark propagator in Eq. (51) by helicity,

$$
S_{\text {ret }}=\left[\begin{array}{cc}
0 & S_{\text {ret }}^{L} \\
S_{\text {ret }}^{R} & 0
\end{array}\right]
$$

We will focus on the right-handed part. At leading order one can write it as

$$
S_{\text {ret }}^{R}(P)=\frac{i}{2 p}\left[\frac{v v^{\dagger}}{p^{0}+E_{\mathbf{p}}+i \Gamma / 2}+\frac{u u^{\dagger}}{p^{0}-E_{\mathbf{p}}+i \Gamma / 2}\right]
$$

where $E_{\mathbf{p}}=\sqrt{p^{2}+m_{\infty}^{2}}$ is the quasi-particle energy. This equation has the same form as in equilibrium but the thermal mass $m_{\infty}^{2}$ and the decay width $\Gamma$ are now outof-equilibrium constants. We have defined $u(\mathbf{p})$ and $v(\mathbf{p})$ to be the eigenvectors of $\boldsymbol{\sigma} \cdot \hat{\mathbf{p}}$ with positive and negative eigenvalues. Their normalization is $v^{\dagger} v=u^{\dagger} u=2 p$ and 


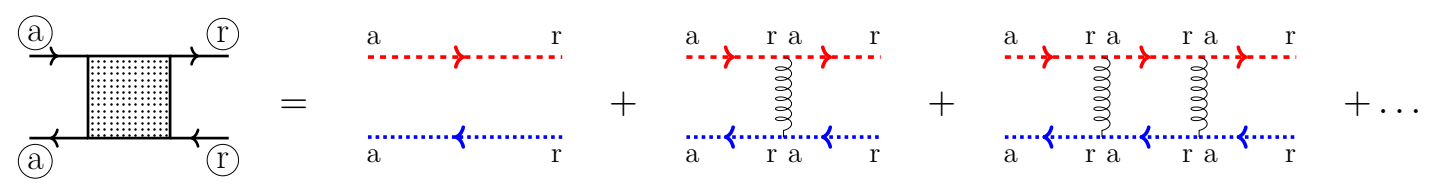

FIG. 18. (Color online) Leading order diagrams contributing to $S_{\text {aarr }}$.

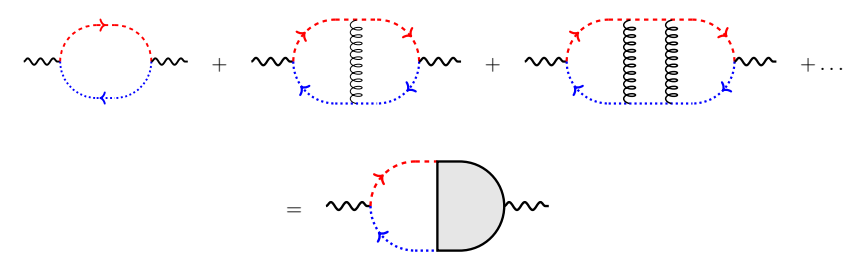

FIG. 19. (Color online) The LPM diagrams that contribute at leading order to the photon polarization tensor $\Pi_{12}^{\gamma}$. Red propagators are $S_{a r}$ and blue propagators are $S_{r a}$.

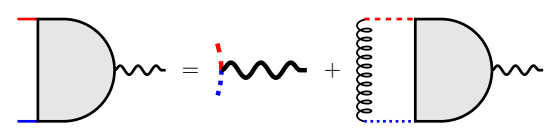

FIG. 20. (Color online) Procedure for summing up the diagrams in Fig. 19 .
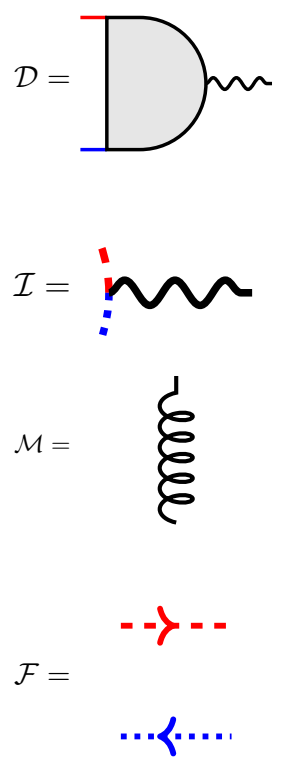

FIG. 21. (Color online) Definition of the quantities in Eq. 68. they obey $v v^{\dagger}=p-\boldsymbol{\sigma} \cdot \mathbf{p}$ and $u u^{\dagger}=p+\boldsymbol{\sigma} \cdot \mathbf{p}$. The first term in Eq. 70 which has $\operatorname{Re} p^{0}<0$ at the pole describes a left-handed antiquark while the second term describes a right-handed quark.

For simplicity we consider the case when $p^{0}>0$ so only the second term in Eq. 70 contributes. At each gluon vertex we get a factor

$$
u^{\dagger}(\mathbf{p}) \sigma^{\mu} u(\mathbf{p})=2(p, \mathbf{p}) \approx 2 P^{\mu}
$$

where the spin indices $u$ come from the adjacent propagators. The gluon rungs then give

$$
\begin{aligned}
\mathcal{M} & =-4 g^{2} C_{F} P_{\mu}\left(K_{\nu}+P_{\nu}\right) G_{r r}^{\mu \nu}(Q) \\
& \approx-4 g^{2} C_{F} p^{z}\left(k+p^{z}\right) \hat{K}_{\mu} \hat{K}_{\nu} \operatorname{Re} G_{r r}^{\mu \nu}(Q) .
\end{aligned}
$$

The loop momentum $P^{\mu}$ is nearly collinear with the photon momentum $K^{\mu}$ so only the real and symmetric part of $G_{r r}$ contributes. Here $\widehat{K}^{\mu}=(1,0,0,1)$.

Using $S_{\mathrm{adv}}^{R}=-S_{\mathrm{ret}}^{R *}$ the pinching pole contribution is easily evaluated to be

$$
\begin{aligned}
\int \frac{d p^{0}}{2 \pi} \mathcal{F}(P ; K) & =\int \frac{d p^{0}}{2 \pi} S_{\mathrm{adv}}^{R}(K+P) S_{\mathrm{ret}}^{R}(P) \\
& =\frac{1}{4 p^{z}\left(p^{z}+k\right)[\Gamma+i \delta E]}
\end{aligned}
$$

where

$$
\delta E=k^{0}+E_{\mathbf{p}} \operatorname{sgn}\left(p^{z}\right)-E_{\mathbf{p}+\mathbf{k}} \operatorname{sgn}\left(p^{z}+k\right)
$$

is of order $g^{2}$. When $p^{0}<0$ one gets similar expressions.

We can now assemble all the pieces in Eq. (68). The quark decay width, Eq. 48, can be written as

$$
\Gamma=\int \frac{d^{2} q_{\perp}}{(2 \pi)^{2}} \mathcal{C}\left(\mathbf{q}_{\perp}\right)
$$

with

$\mathcal{C}\left(\mathbf{q}_{\perp}\right)=g^{2} C_{F} \int \frac{d q_{0} d q_{z}}{(2 \pi)^{2}} 2 \pi \delta\left(q_{0}-q_{z}\right) \operatorname{Re} G_{r r}(Q)^{\mu \nu} \hat{K}_{\mu} \hat{K}_{\nu}$

This collision kernel also describes the gluon rungs. Defining

$$
\widetilde{f}^{\mu}(\mathbf{p})=-4 p^{z}\left(p^{z}+k\right) \int \frac{d p^{0}}{2 \pi} \mathcal{F} \mathcal{D}^{\mu}
$$


one gets that

The production rate of photons with momentum $\mathbf{k}$ is

$\sigma^{\mu}=i \delta E \widetilde{f}^{\mu}(\mathbf{p})+\int \frac{d^{2} q_{\perp}}{(2 \pi)^{2}} \mathcal{C}\left(\mathbf{q}_{\perp}\right)\left[\widetilde{f}^{\mu}(\mathbf{p})-\widetilde{f}^{\mu}\left(\mathbf{p}+\mathbf{q}_{\perp}\right)\right]$

$$
k \frac{d R}{d^{3} k}=\frac{3 Q^{2} \alpha_{E M}}{4 \pi^{2}} \int \frac{d^{3} p}{(2 \pi)^{3}} F(P+K)[1-F(P)] \frac{p^{z 2}+\left(p^{z}+k\right)^{2}}{2 p^{z 2}\left(p^{z}+k\right)^{2}} \mathbf{p}_{\perp} \cdot \operatorname{Re} \mathbf{f}(\mathbf{p} ; \mathbf{k})
$$

as can be seen by using Eq. 67, Eq. (79) and evaluating the trace of the quark loop. Here $\mathbf{f}$ is the transverse part of $\widetilde{f}$ without the Pauli matrix. The new factors in $p^{z}$ and $k$ come from summing over the physical polarization of the photon 20]. Furthermore $Q$ is defined by

$$
Q^{2} e^{2}=\sum_{\text {flavour }} q^{2}
$$

where we sum over the different flavours of light quarks. As explained above, $F$ is the momentum distribution of quarks including Fermi suppression for outgoing quarks,

$$
F(P)=f_{q}(\mathbf{p}) \theta\left(p^{0}\right)+\left(1-f_{\bar{q}}(-\mathbf{p})\right) \theta\left(-p^{0}\right)
$$

Finally $p^{0}=\left(-k^{0}+E_{\mathbf{p}} \operatorname{sgn}\left(p^{z}\right)+E_{\mathbf{p}+\mathbf{k}} \operatorname{sgn}\left(p^{z}+k\right)\right) / 2$.

In this expression $\mathbf{f}$ satisfies a Boltzmann-like integral equation

$\mathbf{p}_{\perp}=i \delta E \mathbf{f}\left(\mathbf{p}_{\perp}\right)+\int \frac{d^{2} q_{\perp}}{(2 \pi)^{2}} \mathcal{C}\left(\mathbf{q}_{\perp}\right)\left[\mathbf{f}\left(\mathbf{p}_{\perp}\right)-\mathbf{f}\left(\mathbf{p}_{\perp}+\mathbf{q}_{\perp}\right)\right]$.

Here $\mathbf{f}\left(\mathbf{p}_{\perp} ; p^{z}, \mathbf{k}\right)$ is an analog of the density of hard quarks with transverse momentum $\mathbf{p}_{\perp}$ that emit a photon with momentum $\mathbf{k}$. The term $i \delta E \mathbf{f}$ works like a time derivative in momentum space. The integral describes the change in transverse momentum of the quarks through the exchange of soft gluons with the medium. The gain term comes from the gluon rungs while the loss term comes from the quark decay width. Eqs. 26) and (82) agree with the abelian limit of the results of [23] where a kinetic theory of quarks and gluons was used to study the LPM effect in a perhaps more heuristic way.

In an isotropic plasma, $f(\mathbf{p})=f(p)$, one gets a simple expression for the collision kernel $\mathcal{C}\left(\mathbf{q}_{\perp}\right)$ by using a sum rule [23, 42]. This special case is relevant for the bulk viscous correction to photon production. Specifically,

$$
\mathcal{C}\left(\mathbf{q}_{\perp}\right)=g^{2} C_{F} \Omega\left[\frac{1}{\mathbf{q}_{\perp}^{2}}-\frac{1}{\mathbf{q}_{\perp}^{2}+m_{D}^{2}}\right] .
$$

Here

$$
\Omega=\frac{\int_{0}^{\infty} d p p^{2}\left[2 N_{f} f_{q}\left(1-f_{q}\right)+2 N_{c} f_{g}\left(1+f_{g}\right)\right]}{-\int_{0}^{\infty} d p p^{2} \frac{d}{d p}\left[2 N_{f} f_{q}+2 N_{c} f_{g}\right]}
$$

characterizes the occupation density of soft gluons. Furthermore,

$$
m_{D}^{2}=\frac{g^{2}}{\pi^{2}} \int_{0}^{\infty} d p p\left[2 N_{f} f_{q}(p)+2 N_{c} f_{g}(p)\right]
$$

is a non-equilibrium Debye mass. In the isotropic case the LPM effect only depends on $\Omega, m_{D}^{2}$ and $m_{\infty}^{2}$, the non-equilibrium mass of hard quarks, along with the momentum distribution $F$ from Eq. 81).

In an anisotropic plasma the collision kernel in Eq. (76) and the quark decay width in Eq. 75 are divergent, because of the gauge field instabilities discussed previously. For the moment, the simplest solution is to impose that the anisotropy is small enough for the divergences to be subleading in the coupling. At leading order only $g^{2} T \lesssim Q \lesssim g T$ contributes to the kernel so one demands that the divergence takes place at the ultrasoft scale of $Q \lesssim g^{2} T$. For the momentum distribution in Eq. (9) this leads to $|\xi| \lesssim g^{2}$. We illustrate this with the $\alpha$ collective mode of Ref. 30. In that case, the divergence in the quark decay width comes from terms like

$$
\int_{g^{2} T}^{g T} d^{4} Q G_{\mathrm{ret}} G_{\mathrm{adv}} \sim \int_{g^{2} T}^{g T} \frac{d^{4} Q}{\left(\mathbf{q}^{2}+m_{\alpha}^{2}\right)^{2}+\left(\frac{\pi q^{0}}{4 q} m_{D}^{2}\right)^{2}}
$$

where $G_{\mathrm{adv}}=G_{\mathrm{ret}}^{*}$ and we have taken the limit $q^{0} \rightarrow 0$ where possible. At leading order in $\xi$, the static limit of the $\alpha$ self-energy component is 30 .

$$
m_{\alpha}^{2}=-\frac{\xi}{6}\left(1+\cos 2 \theta_{n}\right) m_{D}^{2} .
$$

It depends on the angle between the gluon momentum and the direction of the anisotropy, $\theta_{n}$. For $\xi>0, m_{\alpha}^{2}$ is negative which leads to divergences. Clearly, the divergence is at $q \lesssim g^{2} T$ if $\xi \lesssim g^{2}$.

\section{CONCLUSION}

The quark-gluon plasma created in heavy-ion collisions deviates from local thermal equilibrium. To understand how the plasma radiates photons one needs to include the effects of these deviations. This means analyzing photon production in a non-equilibrium plasma. Understanding the effect of viscosity on photons could in turn be used to extract the transport coefficients of QGP from photonic observables.

In this work we have studied photon production through bremsstrahlung and pair annihilation in a non- 
equilibrium QGP. Using field theory, we derived integral equations describing these channels and the LPM effect: Eqs. 82 and 76 . Along the way, we showed that the resummed $r r$ propagator of gluons in an anisotropic plasma has an imaginary part that does not contribute at leading order but could be important at higher order. We also derived a simple expression for the $r r$ propagor of hard, on-shell quarks. Finally, we presented a way of summing up ladder diagrams without using the KMS condition which is only true in thermal equilibrium. Our derivation of the integral equation is valid for low anisotropy. In the special case of an isotropic plasma the integral equation only depends on three non-equilibrium constants.

To solve the integral equation one needs to assume some momentum distribution $f(\mathbf{p})$. Work is ongoing on evaluating the bulk and shear viscous corrections to photon production using $f(\mathbf{p})$ derived from kinetic theory. In the future, these ideas could be used to analyze jets in a non-equilibrium plasma and to extend this work to higher anisotropy. This might allow for the extraction of transport coefficients of QGP from jet observables.

\section{ACKNOWLEDGMENTS}

We thank Stanisław Mrówczyński for useful discussions. This work was supported in part by the Natural Sciences and Engineering Research Council of Canada. S. H. acknowledges a scholarship from the Department of Physics of McGill University, and C. G. acknowledges support from the Canada Council for the Arts through its Killam Research Fellowship program.
[1] Barbara V. Jacak and Berndt Muller, "The exploration of hot nuclear matter," Science 337, 310-314 (2012).

[2] See, for example, Charles Gale, Sangyong Jeon, and Bjoern Schenke, "Hydrodynamic Modeling of Heavy-Ion Collisions," Int. J. Mod. Phys. A28, 1340011 (2013), arXiv:1301.5893 [nucl-th], and references therein.

[3] See, for example, Ulrich Heinz and Raimond Snellings, "Collective flow and viscosity in relativistic heavy-ion collisions," Ann. Rev. Nucl. Part. Sci. 63, 123-151 (2013), arXiv:1301.2826 [nucl-th], and references therein.

[4] Gojko Vujanovic, Clint Young, Bjoern Schenke, Ralf Rapp, Sangyong Jeon, and Charles Gale, "Dilepton emission in high-energy heavy-ion collisions with viscous hydrodynamics," Phys. Rev. C89, 034904 (2014). arXiv:1312.0676 [nucl-th].

[5] Jean-François Paquet, Chun Shen, Gabriel S. Denicol, Matthew Luzum, Bjoern Schenke, Sangyong Jeon, and Charles Gale, "Production of photons in relativistic heavy-ion collisions," Phys. Rev. C93, 044906 (2016), arXiv:1509.06738 [hep-ph]

[6] R. Baier, H. Nakkagawa, A. Niegawa, and K. Redlich, "Production rate of hard thermal photons and screening of quark mass singularity," Z. Phys. C53, 433-438 (1992)

[7] Joseph I. Kapusta, P. Lichard, and D. Seibert, "Highenergy photons from quark - gluon plasma versus hot hadronic gas," Phys. Rev. D44, 2774-2788 (1991)], [Erratum: Phys. Rev.D47,4171(1993)].

[8] M. Strickland, "Thermal photons and dileptons from nonequilibrium quark - gluon plasma," Phys. Lett. B331, 245-250 (1994)

[9] R. Baier, M. Dirks, K. Redlich, and D. Schiff, "Thermal photon production rate from nonequilibrium quantum field theory," Phys. Rev. D56, 2548-2554 (1997), arXiv:hep-ph/9704262 [hep-ph]

[10] F. Gelis, H. Niemi, P. V. Ruuskanen, and S. S. Rasanen, "Photon production from nonequilibrium QGP in heavy ion collisions," Ultra-relativistic nucleus-nucleus collisions. Proceedings, 17th International Conference, Quark Matter 2004, Oakland, USA, January 11-17, 2004, J. Phys. G30, S1031-S1036 (2004), arXiv:nucl-th/0403040

\section{[nucl-th]}

11] Bjoern Schenke and Michael Strickland, "Photon production from an anisotropic quark-gluon plasma," Phys. Rev. D76, 025023 (2007), arXiv:hep-ph/0611332 [hepph].

[12] Maxime Dion, Jean-François Paquet, Bjoern Schenke, Clint Young, Sangyong Jeon, and Charles Gale, "Viscous photons in relativistic heavy ion collisions," Phys. Rev. C84, 064901 (2011), arXiv:1109.4405 [hep-ph].

[13] Chun Shen, Jean-François Paquet, Ulrich Heinz, and Charles Gale, "Photon Emission from a Momentum Anisotropic Quark-Gluon Plasma," Phys. Rev. C91, 014908 (2015), arXiv:1410.3404 [nucl-th]

[14] Sigtryggur Hauksson, Chun Shen, Sangyong Jeon, and Charles Gale, "Bulk viscous corrections to photon production in the quark-gluon plasma," in 8th International Conference on Hard and Electromagnetic Probes of Highenergy Nuclear Collisions: Hard Probes 2016 (HP2016) Wuhan, Hubei, China, September 23-27, 2016 (2016) arXiv:1612.05517 [nucl-th]

[15] P. Aurenche, F. Gelis, and H. Zaraket, "LandauPomeranchuk-Migdal effect in thermal field theory," Phys. Rev. D62, 096012 (2000), arXiv:hep-ph/0003326 [hep-ph].

[16] L. D. Landau and I. Pomeranchuk, "Limits of applicability of the theory of bremsstrahlung electrons and pair production at high-energies," Dokl. Akad. Nauk Ser. Fiz. 92, 535-536 (1953).

[17] L. D. Landau and I. Pomeranchuk, "Electron cascade process at very high-energies," Dokl. Akad. Nauk Ser. Fiz. 92, 735-738 (1953).

[18] Arkady B. Migdal, "Quantum kinetic equation for multiple scattering," Dokl. Akad. Nauk Ser. Fiz. 105, 77 (1955).

[19] Arkady B. Migdal, "Bremsstrahlung and pair production in condensed media at high-energies," Phys. Rev. 103, 1811-1820 (1956).

[20] Peter Brockway Arnold, Guy D. Moore, and Laurence G. Yaffe, "Photon emission from ultrarelativistic plasmas," JHEP 11, 057 (2001), arXiv:hep-ph/0109064 [hep-ph],

[21] Peter Brockway Arnold, Guy D. Moore, and Lau- 
rence G. Yaffe, "Photon emission from quark gluon plasma: Complete leading order results," JHEP 12, 009 (2001), arXiv:hep-ph/0111107 [hep-ph]

[22] Ryogo Kubo, "Statistical mechanical theory of irreversible processes. 1. General theory and simple applications in magnetic and conduction problems," J. Phys. Soc. Jap. 12, 570-586 (1957); Paul C. Martin and Julian S. Schwinger, "Theory of many particle systems. 1." Phys. Rev. 115, 1342-1373 (1959)

[23] Peter Brockway Arnold, Guy D. Moore, and Laurence G. Yaffe, "Effective kinetic theory for high temperature gauge theories," JHEP 01, 030 (2003), arXiv:hep$\mathrm{ph} / 0209353$ [hep-ph]

[24] Michel Le Bellac, Thermal Field Theory (Cambridge University Press, 2011).

[25] Kuang-chao Chou, Zhao-bin Su, Bai-lin Hao, and $\mathrm{Lu} \mathrm{Yu,}$ "Equilibrium and Nonequilibrium Formalisms Made Unified," Phys. Rept. 118, 1 (1985).

[26] L. V. Keldysh, "Diagram technique for nonequilibrium processes," Zh. Eksp. Teor. Fiz. 47, 1515-1527 (1964), [Sov. Phys. JETP20,1018(1965)].

[27] Stanislaw Mrowczynski and Ulrich W. Heinz, "Towards a relativistic transport theory of nuclear matter," Annals Phys. 229, 1-54 (1994).

[28] E. Calzetta and B. L. Hu, "Nonequilibrium Quantum Fields: Closed Time Path Effective Action, Wigner Function and Boltzmann Equation," Phys. Rev. D37, 2878 (1988)

[29] Juergen Berges, "Introduction to nonequilibrium quantum field theory," Proceedings, 9th Hadron Physics and 7th Relativistic Aspects of Nuclear Physics (HADRONRANP 2004): A Joint Meeting on $Q C D$ and $Q G P$ : Rio de Janeiro, Brazil, March 28-April 3, 2004, AIP Conf. Proc. 739, 3-62 (2005), [,3(2004)], arXiv:hep-ph/0409233 [hep-ph]

[30] Paul Romatschke and Michael Strickland, "Collective modes of an anisotropic quark gluon plasma," Phys. Rev. D68, 036004 (2003), arXiv:hep-ph/0304092 [hep-ph]

[31] Stanislaw Mrowczynski, Bjoern Schenke, and Michael Strickland, "Color instabilities in the quarkgluon plasma," Phys. Rept. 682, 1-97 (2017) arXiv:1603.08946 [hep-ph]

[32] Carsten Greiner and Stefan Leupold, "Interpretation and resolution of pinch singularities in nonequilibrium quantum field theory," Eur. Phys. J. C8, 517-522 (1999), arXiv:hep-ph/9804239 [hep-ph].

[33] Julien Serreau, "Out-of-equilibrium electromagnetic radiation," JHEP 05, 078 (2004), arXiv:hep-ph/0310051 [hep-ph]

[34] P. Aurenche, F. Gelis, R. Kobes, and H. Zaraket, "Bremsstrahlung and photon production in thermal QCD," Phys. Rev. D58, 085003 (1998), arXiv:hep$\mathrm{ph} / 9804224$ [hep-ph]

[35] Stanislaw Mrowczynski and Markus H. Thoma, "Hard loop approach to anisotropic systems," Phys. Rev. D62, 036011 (2000), arXiv:hep-ph/0001164 [hep-ph].

[36] Mohammad Nopoush, Yun Guo, and Michael Strickland, "The static hard-loop gluon propagator to all orders in anisotropy," (2017), arXiv:1706.08091 [hep-ph]

[37] J. I. Kapusta and Charles Gale, Finite-temperature field theory: Principles and applications (Cambridge University Press, 2011).

[38] Palash B. Pal, "Representation-independent manipulations with Dirac spinors," (2007), arXiv:physics/0703214 [physics.ed-ph],

[39] Enke Wang and Ulrich W. Heinz, "A Generalized fluctuation dissipation theorem for nonlinear response functions," Phys. Rev. D66, 025008 (2002), arXiv:hepth/9809016 [hep-th]

[40] Kiminad A. Mamo and Ho-Ung Yee, "Spin polarized photons from an axially charged plasma at weak coupling: Complete leading order," Phys. Rev. D93, 065053 (2016), arXiv:1512.01316 [hep-ph].

[41] Sangyong Jeon, "Hydrodynamic transport coefficients in relativistic scalar field theory," Phys. Rev. D52, 35913642 (1995), arXiv:hep-ph/9409250 [hep-ph].

[42] P. Aurenche, F. Gelis, and H. Zaraket, "A Simple sum rule for the thermal gluon spectral function and applications," JHEP 05, 043 (2002), arXiv:hep-ph/0204146 [hep-ph] 